\title{
Optical excitation of atomic force microscopy cantilever for accurate spectroscopic measurements
}

\author{
Yoichi Miyahara ${ }^{1,2,3^{*}+}$ (D), Harrisonn Griffin ${ }^{1 \dagger}$, Antoine Roy-Gobeil ${ }^{1}$, Ron Belyansky ${ }^{1}$, Hadallia Bergeron', \\ José Bustamante ${ }^{1,3}$ and Peter Grutter ${ }^{1}$
}

\author{
*Correspondence: \\ yoichi.miyahara@txstate.edu \\ †Yichi Miyahara and Harrisonn \\ Griffin contributed equally to this \\ work. \\ ${ }^{1}$ Department of Physics, McGill \\ University, 3600 rue University, H3A \\ 2T8 Montreal, Canada \\ ${ }^{2}$ Department of Physics, Texas State \\ University, 601 University Drive, \\ 78666 San Marcos, USA \\ Full list of author information is \\ available at the end of the article
}

\begin{abstract}
Reliable operation of frequency modulation mode atomic force microscopy (FM-AFM) depends on a clean resonance of an AFM cantilever. It is recognized that the spurious mechanical resonances which originate from various mechanical components in the microscope body are excited by a piezoelectric elemen that is intended for exciting the AFM cantilever oscillation and these spurious resonance modes cause the serious undesirable signal artifacts in both frequency shift and dissipation signals. We present an experimental setup to excite only the oscillation of the AFM cantilever in a fiber-optic interferometer system using optical excitation force. While the optical excitation force is provided by a separate laser light source with a different wavelength (excitation laser $: \lambda=1310 \mathrm{~nm}$ ), the excitation laser light is still guided through the same single-mode optical fiber that guides the laser light (detection laser : $\lambda=1550 \mathrm{~nm}$ ) used for the interferometric detection of the cantilever deflection. We present the details of the instrumentation and its performance. This setup allows us to eliminate the problems associated with the spurious mechanical resonances such as the apparent dissipation signal and the inaccuracy in the resonance frequency measurement.
\end{abstract}

Keywords: Atomic force microscopy, Frequecy modulation mode atomic force microscopy, Fiber optic interferometer, Optomechanical coupling

\section{Introduction}

Reliable operation of frequency modulation mode atomic force microscopy (FM-AFM) depends on a clean resonance of an AFM cantilever. It is recognized that the spurious mechanical resonances originating from the various mechanical components of the microscope body are excited inadvertently by a piezoelectric element [1] that is intended for exciting the AFM cantilever oscillation and these spurious resonances cause the serious undesirable signal artifacts in both frequency shift and dissipation signals [2, 3].

The additional frequency-dependent amplitude response causes a crosstalk between the dissipation and frequency shift signals, resulting in artifact signal in dissipation channel which misleads the interpretation of the tip-sample interaction physics. Although such dissipation artifacts could be corrected for by off-line signal processing [3], the additional frequency-dependent phase responses near the cantilever's resonance frequency

(c) The Author(s). 2020 licensee Springer on behalf of EPJ. This is an Open Access article distributed under the terms of the Creative Commons Attribution License (http://creativecommons.org/licenses/by/4.0), which permits unrestricted use, distribution, and reproduction in any medium, provided the original work is properly credited. 
can cause the deviation of the oscillation frequency from the cantilever's resonance frequency for the cantilevers with low quality (Q) factor [2] which makes quantitative measurement of tip-sample interaction challenging. Even in the case of the high-Q cantilever, excessive amount of phase changes due to spurious resonances can cause an interruption of the self oscillation by breaking the phase matching condition, resulting in the loss of distance feedback signal. For this reason, it is important to develop a cantilever excitation scheme which can eliminate the excitation of the spurious mechanical resonances. While the optical excitation of AFM cantilever oscillation has been adopted mainly for the operation in liquid in which case the quality factor is strongly damped [4], it is also widely recognized that the effects of spurious resonances are important at low temperature in high vacuum environment [3] where the Q-factors of spurious resonances are enhanced substantially. Here we report an experimental setup for optical AFM cantilever excitation based on an all fiber optic interferometer which is commonly used for low-temperature AFM systems [5]. As this setup requires only one optical fiber which is used for both sensing and actuation, it can be easily adopted to the existing AFMs which employ fiber-optic interferometers.

\section{Methods/Experimental}

Figure 1 shows a diagram of the optical setup. In this setup, two separate laser lights with different wavelengths are used for the detection (Laser diode 1, $1550 \mathrm{~nm}$ DFB laser with an optical isolater, NECSEL) and excitation (Laser diode 2, $1310 \mathrm{~nm}$, LPS-1310FC, Thorlabs) of the AFM cantilever oscillation. The excitation and detection laser lights are combined with a filter wavelength division multiplexer (FWDM, FWDM-1513, AFW Technology) and launched into a single-mode optical fiber (SMF-28e) [6]. The reflected light from the fiber end and cantilever interfere each other and go back the same FWDM. Only the detection laser light can pass the FWDM and reach a photodiode via an optical circulator (CIR-3-15, AFW Technology) [7]. The intensity of the excitation laser is modulated by modulating the drive current with a power combiner (PRSC-2050, Mini Circuits). The drive current for the detection laser is modulated by a radio frequency signal (several hundred MHz) to suppress stray interferences [8].

We coated the cleaved optical fiber end to increase the back reflection to improve the detection sensitivity of the interferometer [9]. The $\mathrm{TiO}_{2}$ solution used for fiber coating was prepared using a solution of titanium-(IV)-2-ethylhexoxide (Sigma-Aldrich) diluted with xylene. The cleaved fiber end was dipped $1 \mathrm{~cm}$ into the solution, then passed through a propane torch for less than one second for flash annealing. Optimal flash parameters, such as placement of fiber in the flame and time in flame were determined via trial and error. The reflectivity of the fiber was monitored in real time with a photodiode back reflection set up. Successfully annealed fibers would typically have reflectivity between 20 and $30 \%$, with the best fibers having up to $35 \%$. Examples of successfully coated fibers are shown in Fig. 2.

The coated fiber was positioned at the tip end of the cantilever so that the deflection detection sensitivity is maximized. Although this position is not optimal for bolometric actuation [10], it is not critical for the AFM operation in vacuum where the cantilever's Q-factor is high. As will be dicussed later, the magnitude of the excitation force required for achieving several $\mathrm{nm}$ oscillation amplitude is several $\mathrm{pN}$ for the cantilevers with high Q-factor (> 10000) and the optical force of such magnitude can be produced by relatively 


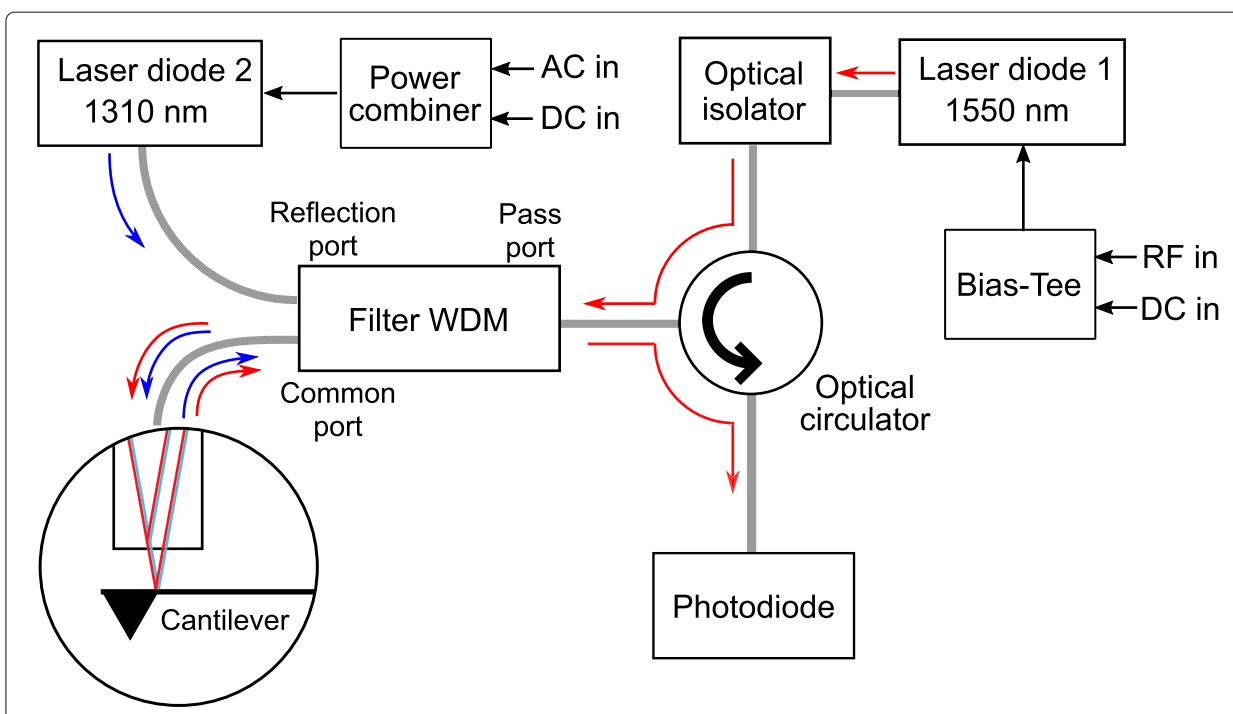

Fig. 1 Schematic diagram of the experimental setup. Two laser lights from Laser diode 1 (1550 nm) and Laser diode $2(1310 \mathrm{~nm})$ are combined with a filter Wavelength Division Multiplexer (FWDM). The combined light is launched into a single-mode optical fiber. While a fraction of the light is reflected at the fiber-vacuum

interface, the rest of the light which comes out of the fiber is reflected on the back side of the cantilever and goes back into the fiber. Only the $1550 \mathrm{~nm}$ laser can pass through the filter WDM and reach the photo diode

small optical power (up to $100 \mu \mathrm{W}$ ) which has negligible effect on the frequency shift noise. The simiplicity of using only a single fiber outweighs the reduced bolotmetric actuation efficiency, particularly, in vacuum/cryogenic temperature environment.

\section{Characterizing optical cavity}

The home built low temperature AFM uses a Fabry-Perot interferometer (FPI) in reflective mode to precisely and accurately measure the distance between the end of the optical fiber and the reflective aluminum coating of the AFM cantilever. An FPI is an optical cavity which is made with two parallel semi-reflective mirrors (mirror 1 and 2) with reflectances $R_{1}$ and $R_{2}$, separated by a cavity length $d$. In our case, the coated fiber and cantilever are the mirror 1 and 2, respectively, and the cavity medium is vacuum. The coated fiber is aligned to the tip end of the cantilever to maximize the deflection measurement sensitivity. Incident light from the detection laser travels down the fiber, where some of the light is internally reflected off of the fiber end, and the rest is injected into the

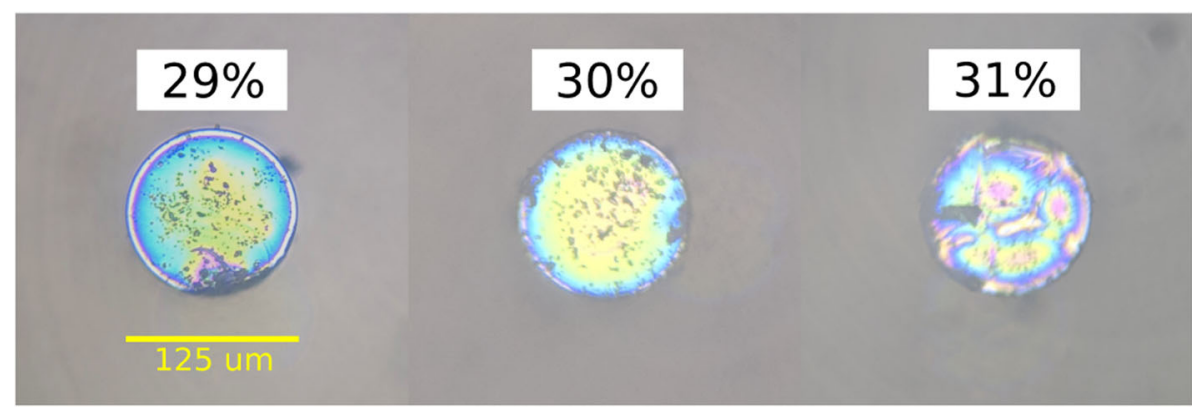

Fig. 2 Three example fibers showing the reflective titanium dioxide coating with varying back reflectance. The fiber has a core diameter of $8 \mu \mathrm{m}$ with a fiber cladding of $125 \mu \mathrm{m}$ radius 
FPI. This light is reflected off of the cantilever where it is then reflected multiple times between the two mirrors of the cavity. Each time the light beam encounters the coated fiber end, it is partially reflected back into the cavity while the rest enters back into the fiber. All the transmitted beams into the fiber together with the initially reflected beam contribute to the interference inside the fiber. The reentered light is guided through the fiber, WDM, and circulator and its intensity, $I_{r}$, is measured by the photodiode (Fig. 1). The FPI lends itself to being more sensitive than other interferometers because of the multiple reflections between the mirror 1 and 2 when the separation between the mirrors is small enough [11-13]. It is important to notice that the multiple reflection also plays an important role for the excitation laser light as it enhances the optical force. We use the FPI model reported in Ref. [14] for the following analysis.

The ratio of the intracavity intensity, $I_{\text {circ }}$, to the intensity incident upon mirror $1, I_{\mathrm{inc}}$, (enhancement factor, $A_{\text {circ }}^{\prime}$ ) is given by Airy distribution,

$$
A_{\text {circ }}^{\prime}=\frac{I_{\text {circ }}}{I_{\text {inc }}}=\frac{1-R_{1}}{\left(1-\sqrt{R_{1} R_{2}}\right)^{2}+4 \sqrt{R_{1} R_{2}} \sin ^{2} \phi} .
$$

where $\phi$ is the single-pass phase shift between the mirrors and can be expressed as

$$
\phi=\frac{2 \pi d}{\lambda}
$$

where $d$ is the distance between two mirrors (cavity length) and $\lambda$ is wavelength. Similarly, the enhancement factor for the total back reflected light with respect to $I_{\text {inc }}$ is given by

$$
\frac{I_{\text {refl }}}{I_{\text {inc }}}=\frac{\left(\sqrt{R_{1}}-\sqrt{R_{2}}\right)^{2}+4 \sqrt{R_{1} R_{2}} \sin ^{2} \phi}{\left(1-\sqrt{R_{1} R_{2}}\right)^{2}+4 \sqrt{R_{1} R_{2}} \sin ^{2} \phi} .
$$

Figure 3a shows the experimentally measured interference fringe and theoretical one given by Eq. 3 assuming $R_{1}=0.33$ and $R_{2}=0.96$. These values are in good agreement with the value obtained from the measured back reflection of the coated fiber $\left(R_{1}=0.30\right)$ and the reflectance of the $\mathrm{Al}$ coated cantilever $R_{2}=0.96$ assuming $100 \%$ collection of the reflected beam from the cantilever. The good agreement between the theory and experiment confirms the validity of the model. For $R_{1}=0.33$ and $R_{2}=0.96$, the enhancement factor, $A_{\text {circ }}^{\prime}$ is found to be 3.5 .

\section{Results and discussion}

Figure 4 shows the frequency responses of an AFM cantilever (NCLR Nanosensors, Al backside coated and Pt tip-side coated) excited by piezo excitation (blue line) and optical excitation (red line). The response by piezo excitation shows spurious resonance modes over the entire $5 \mathrm{kHz}$ frequency range around the cantilever's fundamental resonance frequency. These additional frequency-dependent amplitude and phase responses are not present when the cantilever is optically driven. The optical force does not excite the various mechanical resonances of the microscope body, thus giving us cleaner signals in the frequency shift and dissipation channels. Through removing the artifacts, we can better study the tip-sample interaction physics.

\section{Quantifying optical excitation force}

In order to determine the magnitude of the optical force quantitatively, we use the following relation between the oscillation amplitude and the amplitude of the excitation optical force when the cantilever is excited by harmonic excitation force, $F(t)=F_{\text {opt }} \sin \left(2 \pi f_{0} t\right)$, 

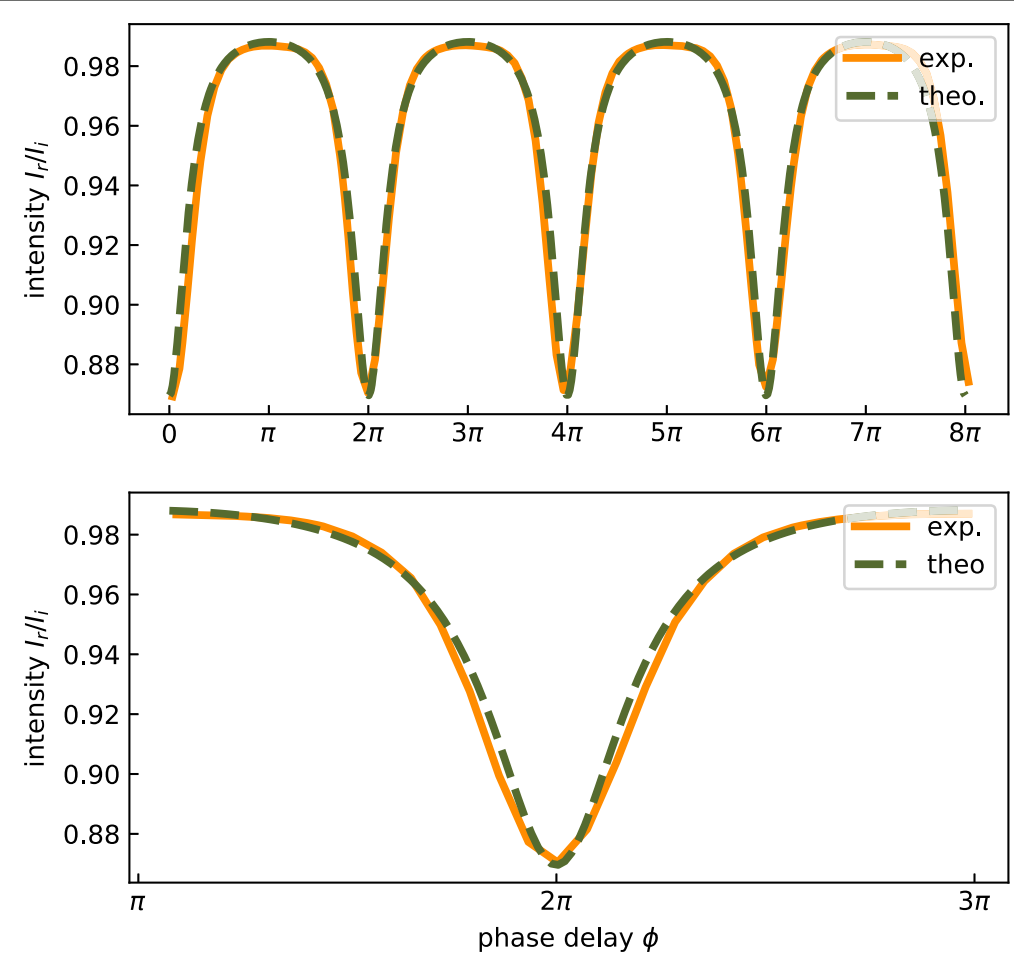

Fig. 3 Theoretical and experimental transfer function of the reflective mode of the Fabry-Perot interferometer. Top shows the measured and theoretical interference response of the cavity as a function of increasing phase delay (i.e. increasing fiber-cantilever separation). This fiber end has a 30\% back reflection. Bottom shows the first full interference peak that occurs as peak intensity as a function of phase delay. The experimental data is plotted with the theoretical fit
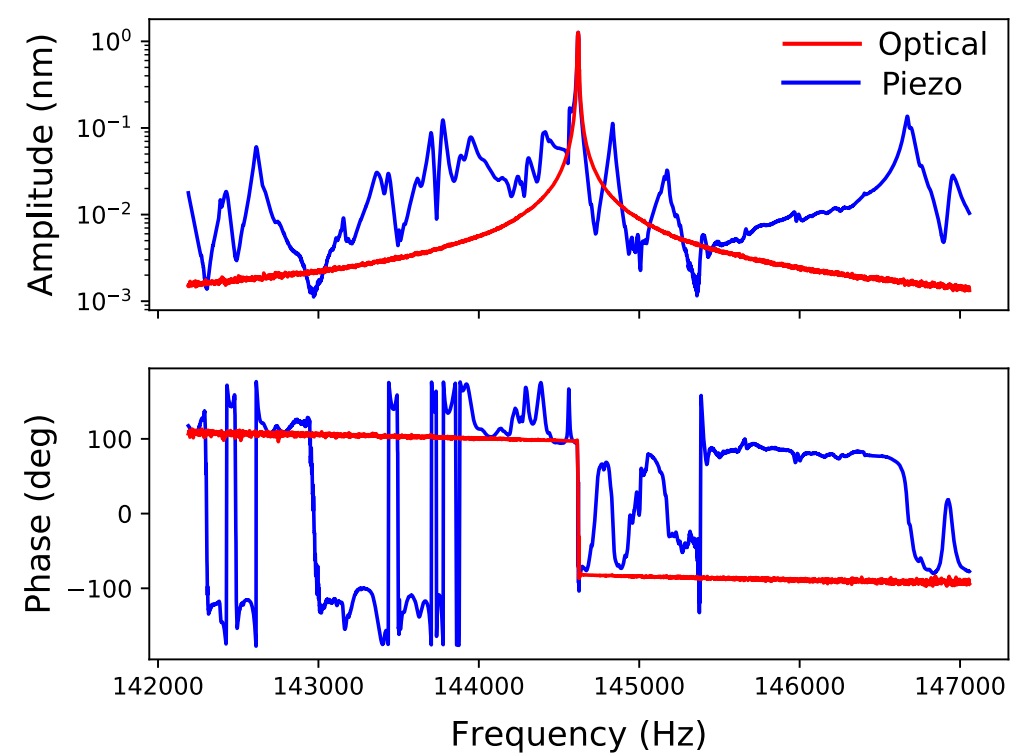

Fig. 4 Amplitude and phase frequency responses of the AFM cantilever excited by optical excitation (red) and piezo excitation (blue) measured at $4.5 \mathrm{~K}$. Note the spurious mechanical resonances in the piezo excitation which arise from vibrations in the microscope body 


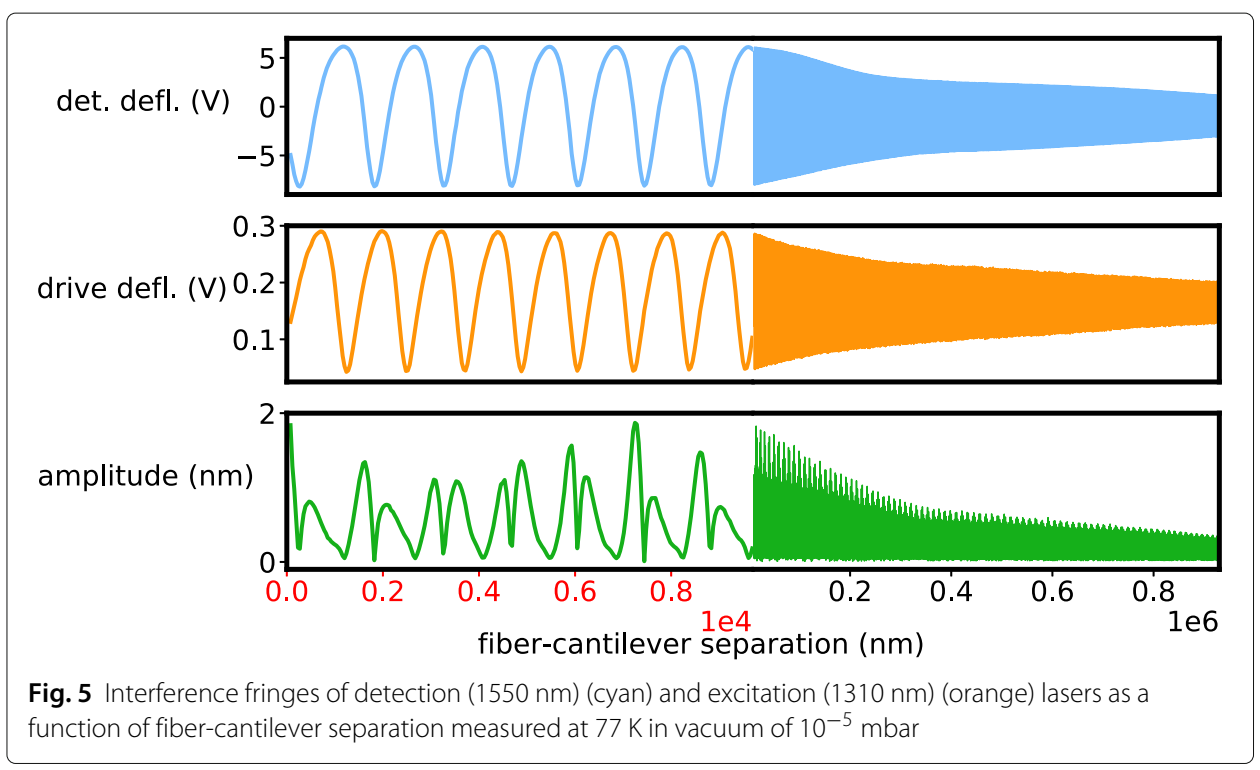

at the resonance frequency, $f_{0}$ :

$$
A=\frac{Q}{k} F_{\text {opt }}
$$

Here $A$ is the amplitude of the oscillation, $Q$ the quality factor, $k$ the spring constant of the cantilever and $F_{\text {opt }}$ the amplitude of the oscillating optical force, We used the Sader method to calibrate the effective spring constant of the fundamental flexural mode of cantilevers [15] and obtained a spring constant $k=19 \mathrm{~N} / \mathrm{m}$ for the cantilever used.

In our interferometer setup, the fiber-cantilever distance can be adjusted by a piezoelectric stick-slip motor with the step size as small as $20 \mathrm{~nm}$. The step size is confirmed to be very uniform over several fringes. As we change the fiber-cantilever distance by stepping the fiber position, we record the signal at the photodiode $V_{\mathrm{PD}}$. The resulting interference fringes show nearly periodic peaks and the peak separation, known to be $\lambda / 2$ from Eq. 3. This well-defined separation allows us to calibrate the horizontal axis that determine the sensitivity of the interferometer.

The interferometer sensitivity $S$ is calculated as the derivative of the measured interferometer output voltage $V_{\mathrm{PD}}$ with respect to the fiber fiber-cantilever distance $z$ [16]: Since the distance between successive peaks is known, we can convert the walker steps $\Delta z$ into a distance by identifying successive peak positions and counting the steps between them. Now we can simply take the derivative of the photodiode signal versus the fiber-cantilever distance, then divide by the walker step $\Delta z$ to obtain the sensitivity $S$. An example of such measurement is shown in Fig. 6.

With the known sensitivity in units of $\mathrm{V} / \mathrm{nm}$, it is straightforward to determine the oscillation amplitude of the cantilever. We measure the ac component of the photodiode signal $V_{\mathrm{PD}}$, and convert its amplitude in Volt to the amplitude in $\mathrm{nm}$ by dividing $V_{\mathrm{PD}}$ by the sensitivity $S$. The typical sensitivity during the experiments is $30 \mathrm{mV} / \mathrm{nm}$. This sensitivity can also be used to calculate the detection noise of the interferometer. By taking a power spectral density of $V_{\mathrm{PD}}$ around the cantilever resonance, we can convert the measured interferometer noise in $\mathrm{V} / \sqrt{\mathrm{Hz}}$ into the detection noise in $\mathrm{fm} / \sqrt{\mathrm{Hz}}$. A typical 

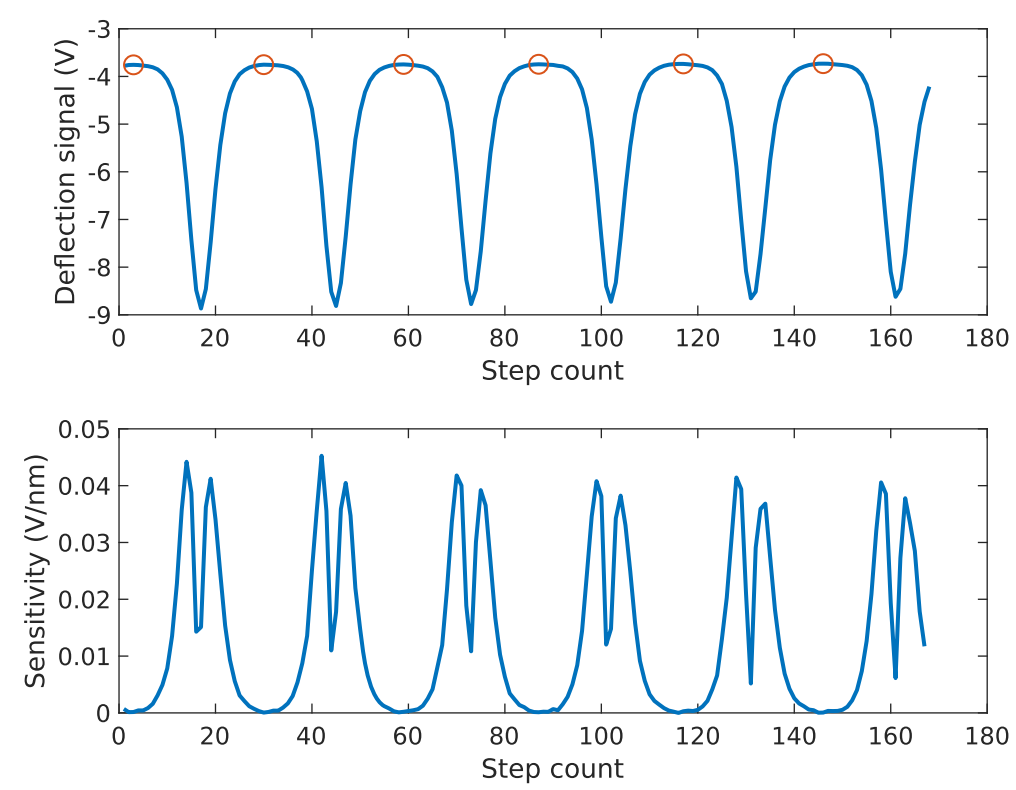

Fig. 6 Top shows the photodiode signal as a function of the number of fiber walker steps as it is retracted from the cantilever. Peaks are indicated via circles, which are then used to determine the number of steps between adjacent fringes. Bottom shows sensitivity $S$ which is calculated by taking the derivative of the photodiode signal and dividing by the average step size between fringes

detection noise at $77 \mathrm{~K}$ is $\approx 50 \mathrm{fm} / \sqrt{\mathrm{Hz}}$ and at $4 \mathrm{~K}$ is $\approx 15 \mathrm{fm} / \sqrt{\mathrm{Hz}}$. The best detection noise we have observed at $4 \mathrm{~K}$ is $7 \mathrm{fm} / \sqrt{\mathrm{Hz}}$.

From the measured $A, Q$ and $k$, we are able to obtain the optical excitation force, $F_{\mathrm{opt}}$ as a function of the amplitude of the modulated optical power by using Eq. 4, as shown on the right hand side axis of Fig. 7. We measured the optical power of the $1310 \mathrm{~nm}$ driving laser with a separate photodiode placed at the $10 \%$ branch of a $90 / 10$ coupler (not shown in Fig. 1) and its modulated amplitude, $P$, with the lock-in amplifier. Figure 7 shows the measured $A$-P relations with four different dc offset current settings. We can see that the oscillation amplitude is just dependent on the amplitude of modulated optical power and does not depend on the dc power. The average slope of the four data sets is $68.4 \mathrm{pN} / \mathrm{mW}$.

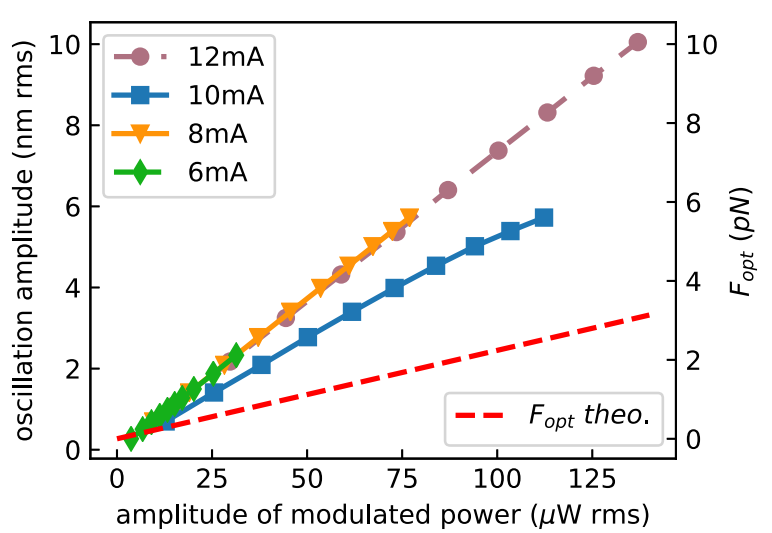

Fig. 7 Cantilever oscillation amplitude as a function of modulation power at varying laser diode currents (solid lines). The right vertical axis indicates the corresponding optical force. The red dashed line shows the theoretical optical scattering force 
Now let us compare the measured driving force with the theoretical optical forces. The theoretical optical scattering force (radiation pressure force), $F_{\text {scat }}$, acting on the AFM cantilever with reflectance $R_{2}$ in a FPI system is given by

$$
F_{\text {scat }}=\frac{2 P_{\text {circ }} R_{2}}{c}=\frac{2 A_{\text {circ }}^{\prime} P_{\text {inc }} R_{2}}{c}
$$

where $P_{\text {circ }}$ is the optical power in the FPI cavity, $P_{\text {inc }}$ the incident optical power and $c$ the speed of light. While the enhancement factor $A_{\text {circ }}^{\prime}$ is in general dependent on the wavelength due to the wavelength-dependence of $R_{2}$, we confirmed $A_{\text {circ }}^{\prime}=3.5$ for the excitation laser $(\lambda=1330 \mathrm{~nm})$ by measuring the interferometer fringes as shown in Fig. 8. The theoretical slope of $F_{\text {scat }}-P_{\text {inc }}$ is therefore $F_{\text {scat }} / P_{\text {inc }}=22.4 \mathrm{pN} / \mathrm{mW}$ for $A_{\text {circ }}^{\prime}=3.5$ and $R_{2}=0.96$. The fact that the experimental optical force $F_{\mathrm{opt}}$ is much larger than the expected optical scattering force $F_{\text {scat }}$ indicates that the photothermal (bolometric) effect plays an important role [11].

\section{Effect of optical driving force on frequency shift noise}

In FM-AFM, the interaction forces between the sample and cantilever are detected as a frequency shift. Since the tip-sample distance is regulated by the frequency shift, a reduction in frequency shift noise will lead to high-resolution FM-AFM images. Additionally, in electrostatic force microscopy with single-electron sensitivity (e-EFM) measurements, achieving a detectable signal is only possible with a reduced frequency shift noise [16, 17]. In the following we will investigate if optically driving the cantilever introduces additional frequency noise. The frequency shift noise measurements were performed at $77 \mathrm{~K}$ with the AFM cantilevers that have spring constants of $k=20 \mathrm{~N} / \mathrm{m}$ and quality factors at $77 \mathrm{~K}$ varying between 15,000 and 50,000. In these experiments, the cantilever is self-excited
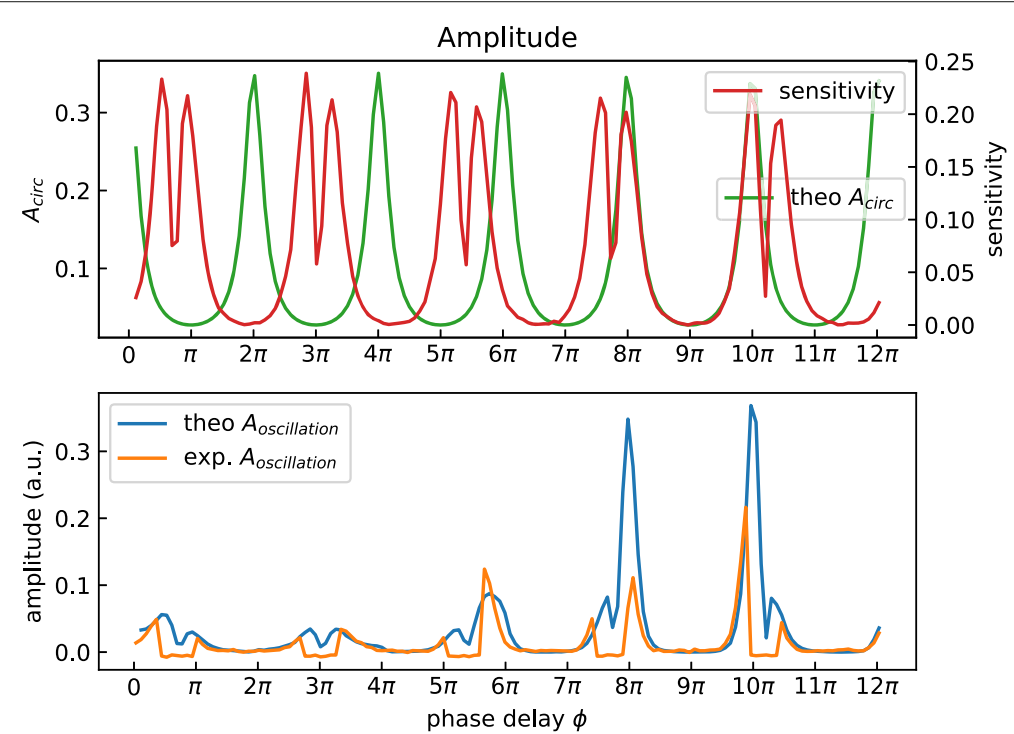

Fig. 8 Top shows the theoretical $A_{\text {circ }}^{\prime}$ and the sensitivity $S$ for a set of interference fringes. Bottom shows the theoretical and experimentally measured oscillation amplitude of the cantilever as a function of phase delay (cavity length). The theoretical amplitude was calculated by multiplying circulating enhancement factor $A_{\text {circ }}^{\prime}$ by sensitivity $S$ 
at its resonant frequency by feeding the deflection signal into the optical excitation system through an oscillation control electronics (easyPLLplus controller, Nanosurf) which consists of a phase shifter and amplitude controller [18].

To see if the average power of the excitation source affected the frequency shift noise of the microscope, we measured the frequency shift noise at various oscillation amplitudes from 0.5 to $10 \mathrm{~nm}$, and increased the average optical power of the excitation laser. The amplitude was kept constant at different average optical powers. The frequency shift measurement was made using a phase-locked loop (PLL) frequency detector (HF2LI with PLL option, Zurich Instruments) with a detection bandwidth of $100 \mathrm{~Hz}$. The frequency shift noise was measured using the root mean square value of the frequency shift and is shown in Fig. 9. As expected from the theory [18], the frequency shift noise increases with decreasing oscillation amplitude, but there is no significant variation in the frequency shift noise at the same oscillation amplitude for varying average optical excitation power.

Since we can precisely adjust our fiber-cantilever separation distance, we are able to choose an operating position on an interference fringe arbitrarily. Typically, we choose a fringe position that maximizes the sensitivity. On a single fringe, we could choose the operating position either on the negative or positive slope side of the fringe. It has been reported that the effective Q-factor is decreased or increased depending on the slope of the interferometer signal due to the optomechanical coupling $[11,19,20]$. For example, by adjusting the fiber-cantilever cavity position to be on either side of a single fringe at the same deflection DC value, we observe the effective Q-factors at the negative and positive slope side to vary as much as 3000 .

To determine if the different effective Q-factor at the negative and positive slope affect the frequency shift noise, we measured the frequency shift noise at various oscillation amplitudes on both the negative and positive slope side of the fringe at the same DC deflection values, as shown in Fig. 10. The results shows negligible difference in frequency shift noise between the negative slope and positive slope side of the fringe.

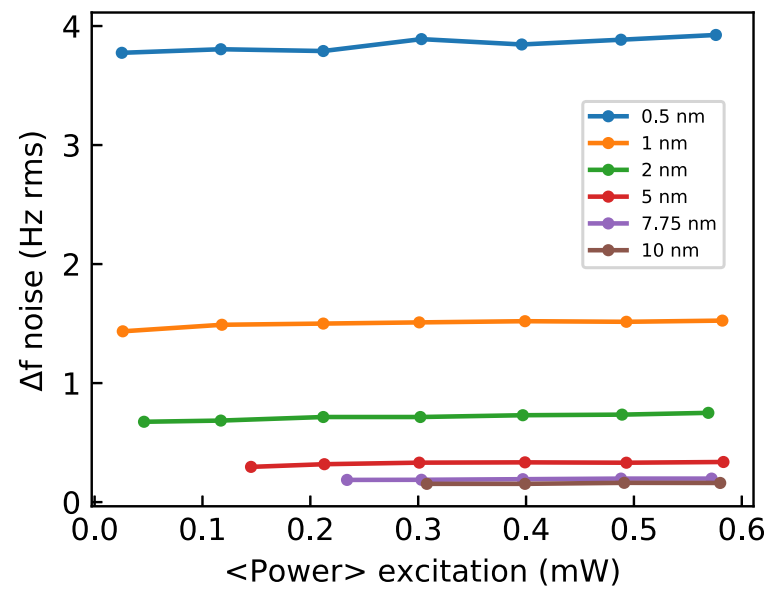

Fig. 9 Frequency shift noise as a function of average optical power of the excitation laser at varying oscillation amplitudes. The frequency shift noise was measured as the root mean square (RMS) of the frequency shift from the lock-in amplifier. The lines are a guide to the eyes 


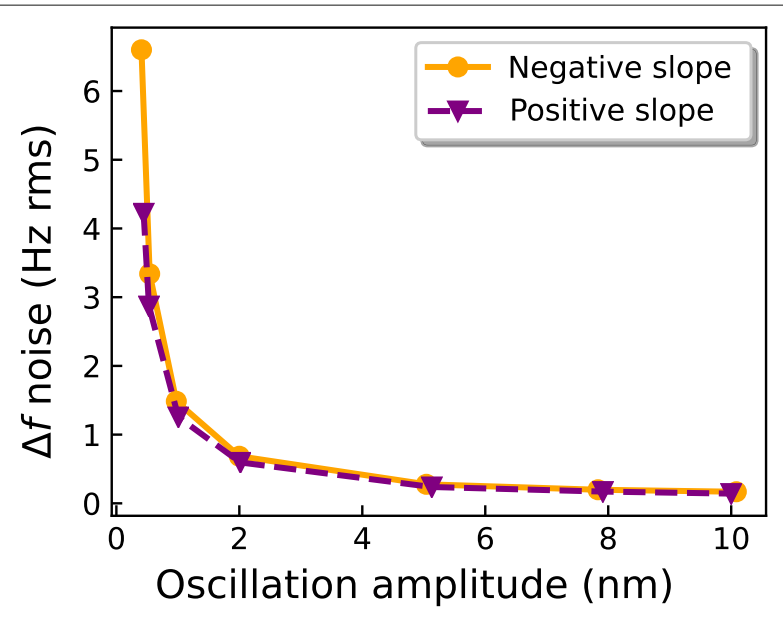

Fig. 10 Frequency shift noise $(\Delta f)$ as a function of oscillation amplitude of cantilever being driven via optical excitation at $77 \mathrm{~K} . \Delta f$ is measured on the negative slope (orange circle) and positive slope (purple triangle) of the interference fringe. The lines are a guide to the eyes

\section{Conclusion}

In conclusion, we have integrated an optical drive cantilever excitation system into our low temperature AFM in a non-invasive way. By using the optical circuit as shown in Fig. 1, we are able to have a two laser detection and excitation system deployed through a single optical fiber. The optical drive reduces spurious mechanical resonances making the AFM suitable to study sensitive tip-sample physics without artifacts from crosstalk in the frequency shift and dissipation channels. The setup can easily be adopted for the existing AFMs which use fiber-optic interferometer.

\section{Authors' contributions}

YM designed and constructed the experimental setup. YM, HG, ARG and JB performed the experiments. YM, HG and ARG analyzed the data. RB and $\mathrm{HB}$ contributed to the preliminary experiments. YM and PG conceived of the study. YM, HG and $P G$ wrote the manuscript. All authors read and approved the final manuscript.

\section{Authors' information}

Correspondence and requests for materials should be addressed to YM (yoichi.miyahara@txstate.edu) or PG (peter.grutter@mcgill.ca).

\section{Funding}

Financial support from The Natural Sciences and Engineering Research Council of Canada and Le Fonds de Recherche du Québec-Nature et Technologies are gratefully acknowledged. JB acknowledges financial support from National Secretary of Higher Education, Science and Technology and Innovation, Ecuador.

\section{Availability of data and materials}

Not applicable.

\section{Competing interests}

The authors declare that they have no competing interests.

\section{Author details}

${ }^{1}$ Department of Physics, McGill University, 3600 rue University, H3A 2T8 Montreal, Canada. ${ }^{2}$ Department of Physics, Texas State University, 601 University Drive, 78666 San Marcos, USA. ${ }^{3}$ Departamento de Fisica, Universidad San Francisco de Quito, Diego de Robles S/N, 170901 Quito, Ecuador.

Received: 31 October 2019 Accepted: 27 January 2020

Published online: 10 February 2020

\section{References}

1. Schäffer TE, Cleveland JP, Ohnesorge F, Walters DA, Hansma PK. Studies of vibrating atomic force microscope cantilevers in liquid. J Appl Phys. 1996;80(7):3622-7. https://doi.org/10.1063/1.363308. 
2. Kobayashi $\mathrm{K}$, Yamada $\mathrm{H}$, Matsushige $\mathrm{K}$. Reduction of frequency noise and frequency shift by phase shifting elements in frequency modulation atomic force microscopy. Rev Sci Inst. 2011;82(3):033702. https://doi.org/10. 1063/1.3557416.

3. Labuda A, Miyahara Y, Cockins L, Grutter PH. Decoupling conservative and dissipative forces in frequency modulation atomic force microscopy. Phys Rev B. 2011;84(12):125433. https://doi.org/10.1103/PhysRevB.84.125433.

4. Labuda A, Kobayashi K, Miyahara Y, Grütter P. Retrofitting an atomic force microscope with photothermal excitation for a clean cantilever response in low Q environments. Rev Sci Inst. 2012;83(May):053703. https://doi.org/ 10.1063/1.4712286.

5. Rugar D, Mamin HJ, Guethner P. Improved fiber-optic interferometer for atomic force microscopy. Appl Phys Lett. 1989:55(25):2588-90. https://doi.org/10.1063/1.101987.

6. Weld DM, Kapitulnik A. Feedback control and characterization of a microcantilever using optical radiation pressure. Appl Phys Lett. 2006;89(16):164102. https://doi.org/10.1063/1.2362598.

7. Rasool HI, Wilkinson PR, Stieg AZ, Gimzewski JK. A low noise all-fiber interferometer for high resolution frequency modulated atomic force microscopy imaging in liquids. Rev Sci Inst. 2010;81(2):023703. https://doi.org/10.1063/1. 3297901.

8. Smith DT, Pratt JR, Howard LP. A fiber-optic interferometer with subpicometer resolution for dc and low-frequency displacement measurement. Rev Sci Inst. 2009;80(3):035105. https://doi.org/10.1063/1.3097187.

9. Subba-Rao V, Sudakar C, Esmacher J, Pantea M, Naik R, Hoffmann PM. Improving a high-resolution fiber-optic interferometer through deposition of a TiO2 reflective coating by simple dip-coating. Rev Sci Inst. 2009;80(11): 115104. https://doi.org/10.1063/1.3244088.

10. Kiracofe D, Kobayashi K, Labuda A, Raman A, Yamada H. High efficiency laser photothermal excitation of microcantilever vibrations in air and liquids. Rev Sci Inst. 2011;82(1):013702. https://doi.org/10.1063/1.3518965.

11. Vogel M, Mooser C, Karrai K, Warburton RJ. Optically tunable mechanics of microlevers. Appl Phys Lett. 2003;83(7): 1337-9. https://doi.org/10.1063/1.1600513.

12. Wilkinson PR, Pratt JR. Analytical model for low finesse, external cavity, fiber Fabry-Perot interferometers including multiple reflections and angular misalignment. Appl Opt. 2011;50(23):4671. https://doi.org/10.1364/AO.50.004671.

13. Von Schmidsfeld A, Reichling M. Controlling the opto-mechanics of a cantilever in an interferometer via cavity loss. Appl Phys Lett. 2015;107(12):2-7. https://doi.org/10.1063/1.4931702.

14. Ismail N, Kores CC, Geskus D, Pollnau M. Fabry-Pérot resonator: spectral line shapes, generic and related Airy distributions, linewidths, finesses, and performance at low or frequency-dependent reflectivity. Opt Express. 2016;24(15):16366. https://doi.org/10.1364/OE.24.016366.

15. Sader JE, Chon JW, Mulvaney P. Calibration of rectangular atomic force microscope cantilevers. Rev Sci Inst. 1999;70(10):3967-9.

16. Roy-Gobeil A. Single-electron charging using atomic force microscopy. PhD thesis: McGill University; 2017.

17. Roy-Gobeil A, Miyahara Y, Bevan KH, Grutter P. Fully Quantized Electron Transfer Observed in a Single Redox Molecule at a Metal Interface. Nano Lett. 2019;19(9):6104-8. https://doi.org/10.1021/acs.nanolett.9b02032.

18. Albrecht TR, Grütter P, Horne D, Rugar D. Frequency modulation detection using high- Q cantilevers for enhanced force microscope sensitivity. J Appl Phys. 1991;69(2):668-73. https://doi.org/10.1063/1.347347.

19. Hölscher $H$, Milde P, Zerweck U, Eng LM, Hoffmann R. The effective quality factor at low temperatures in dynamic force microscopes with Fabry-Pérot interferometer detection. Appl Phys Lett. 2009;94(22):223514. https://doi.org/ 10.1063/1.3149700.

20. Tröger $L$, Reichling M. Quantification of antagonistic optomechanical forces in an interferometric detection system for dynamic force microscopy. Appl Phys Lett. 2010;97(21):213105. https://doi.org/10.1063/1.3509412.

\section{Publisher's Note}

Springer Nature remains neutral with regard to jurisdictional claims in published maps and institutional affiliations.

\section{Submit your manuscript to a SpringerOpen ${ }^{\circ}$ journal and benefit from:}

- Convenient online submission

- Rigorous peer review

- Open access: articles freely available online

- High visibility within the field

- Retaining the copyright to your article

Submit your next manuscript at $\boldsymbol{\triangleright}$ springeropen.com 